For families of SIDS victims, a clear delineation of risk factors, both genetic and environmental, will be instrumental in identifying children who may benefit from therapeutic intervention. Bowers et al. (8) make cogent suggestions for further study and possible screening for this particular genetic risk factor in select populations. It is likely that uncovering mutations in SCN5A and other ion channels will be just the beginning of determining the complex genetic basis for SIDS, and in the years ahead other genetic abnormalities may also be linked to SIDS. Current thinking emphasizes a brain stem neural network maldevelopment hypothesis for this syndrome (19). It is likely that the etiology of SIDS is heterogeneous and may result from the interaction of a number of genetic and environmental factors. It remains to be determined how many cases of SIDS may eventually be linked to mutations predisposing the carrier to cardiac arrhythmia.

\section{Acknowledgments}

This work is supported by NIH grants HD42569 and HL71092 to the author.

Address correspondence to: Jonathan C. Makielski, Department of Medicine, Uni- versity of Wisconsin, 600 Highland Avenue, H6/349, Madison, Wisconsin 53792, USA. Phone: (608) 263-9648; Fax: (608) 2630405; E-mail: jcm@medicine.wisc.edu.

1. Schwartz, P.J. 1976. Cardiac sympathetic innervation and the sudden infant death syndrome. A possible pathogenetic link. Am. J. Med. 60:167-172.

2. Maron, B.J., Clark, C.E., Goldstein, R.E., and Epstein, S.E. 1976. Potential role of QT interval prolongation in sudden infant death syndrome. Circulation. 54:423-430.

3. Keating, M.T., and Sanguinetti, M.C. 2001. Molecular and cellular mechanisms of cardiac arrhythmias. Cell. 104:569-580.

4. Schwartz, P.J., et al. 2000. A molecular link between the sudden infant death syndrome and the longQT syndrome. N. Engl.J. Med. 343:262-267.

5. Ackerman, M.J., et al. 2001. Postmortem molecular analysis of SCN5A defects in sudden infant death syndrome. JAMA. 286:2264-2269.

6. Splawski, I., et al. 2002. Variant of SCN5A sodium channel implicated in risk of cardiac arrhythmia. Science. 297:1333-1336.

7. Tan, B.H., et al. 2005. Common human SCN5A polymorphisms have altered electrophysiology when expressed in Q1077 splice variants. Heart Rhythm. 2:741-747.

8. Plant, L.D., et al. 2006. A common cardiac sodium channel variant associated with sudden infant death in African Americans, SCN5A S1103Y.J. Clin. Invest. 116:430-435. doi:10.1172/JCI25618.

9. Ackerman, M.J., et al. 2004. Spectrum and prevalence of cardiac sodium channel variants among black, white, Asian, and Hispanic individuals: implications for arrhythmogenic susceptibility and Brugada/long QT syndrome genetic testing. Heart
Rhythm. 1:600-607.

10. Makielski, J.C., et al. 2003. A ubiquitous splice variant and a common polymorphism affect heterologous expression of recombinant human SCN5A heart sodium channels. Circ. Res. 93:821-828.

11. Ye, B., Valdivia, C.R., Ackerman, M.J., and Makielski, J.C. 2002. A common human SCN5A polymorphism modifies expression of an arrhythmia causing mutation. Physiol. Genomics. 12:187-193.

12. Isom, L.L. 2001. Sodium channel beta subunits: anything but auxiliary. Neuroscientist. 7:42-54.

13. Adamson, P.B., et al. 2005. The perplexing complexity of cardiac arrhythmias: beyond electrical remodeling. Heart Rhythm. 2:650-659.

14. Nuyens, D., et al. 2001. Abrupt rate accelerations or premature beats cause life-threatening arrhythmias in mice with long-QT3 syndrome. Nat. Med. 7:1021-1027.

15. Skinner, J.R., et al. 2005. Near-miss SIDS due to Brugada syndrome. Arch. Dis. Child. 90:528-529.

16. Schwartz, P.J., et al. 2001. Molecular diagnosis in a child with sudden infant death syndrome. Lancet. 358:1342-1343.

17. Christiansen, M., et al. 2005. Mutations in the HERG $\mathrm{K}^{+}$-ion channel: a novel link between long QT syndrome and sudden infant death syndrome. Am. J. Cardiol. 95:433-434.

18. Wedekind, H., et al. 2005. Sudden infant death syndrome and long QT syndrome: an epidemiological and genetic study. Int. J. Legal Med. doi:10.1107/ s00414-005-0019-0.

19. American Academy of Pediatrics Task Force on Sudden Infant Death Syndrome. 2005. The changing concept of sudden infant death syndrome: diagnostic coding shifts, controversies regarding the sleeping environment, and new variables to consider in reducing risk. Pediatrics. 116:1245-1255.

\title{
Gastrointestinal motility and glycemic control in diabetes: the chicken and the egg revisited?
}

\author{
Christopher K. Rayner and Michael Horowitz
}

University of Adelaide Department of Medicine, Royal Adelaide Hospital, Adelaide, South Australia, Australia.

\begin{abstract}
Upper gastrointestinal dysfunction occurs frequently in diabetes and potentially contributes to both abdominal symptoms and impaired glycemic control; conversely, variations in blood glucose concentration reversibly affect gut motility in humans. In this issue of the JCI, Anitha et al. report apoptosis of rodent enteric neurons under hyperglycemic conditions, both in vitro and in vivo, associated with impaired PI3K activity and preventable by glial cell line-derived neurotrophic factor (see the related article beginning on page 344). These observations add to recent insights gained from animal models regarding the etiology of diabetic gastrointestinal dysfunction, but investigators must strive to translate animal data to human diabetes.
\end{abstract}

Nonstandard abbreviations used: GDNF, glial cell line-derived neurotrophic factor; GLP-1, glucagonlike peptide 1 ; ICC, interstitial cell of Cajal; STZ, streptozotocin.

Conflict of interest: The authors have declared that no conflict of interest exists.

Citation for this article: J. Clin. Invest. 116:299-302 (2006). doi:10.1172/JCI27758.
It is now recognized that disordered gastrointestinal motor and sensory function occur frequently in diabetes mellitus and that this has substantial implications for the morbidity and effective management of patients with this condition. For example, gastric emptying is abnormally delayed in $30-50 \%$ of outpatients with long-stand- ing type 1 or type 2 diabetes, with potential consequences of gastrointestinal symptoms, impaired nutrition, poor glycemic control, and delayed absorption of oral medications (1). However, the magnitude of the delay in gastric emptying is variable and in many cases modest (2). While diabetic gastroparesis is often associated with symptoms such as nausea, vomiting, postprandial fullness, and bloating, the relationship of symptoms to disordered emptying is relatively weak, and some patients are asymptomatic (2). Intestinal transit is also often disturbed in diabetes (rapid or slow) (3), and symptoms such as diarrhea and constipation occur more frequently than in the general population (4).

Disordered gastrointestinal motility in human diabetes has traditionally been 


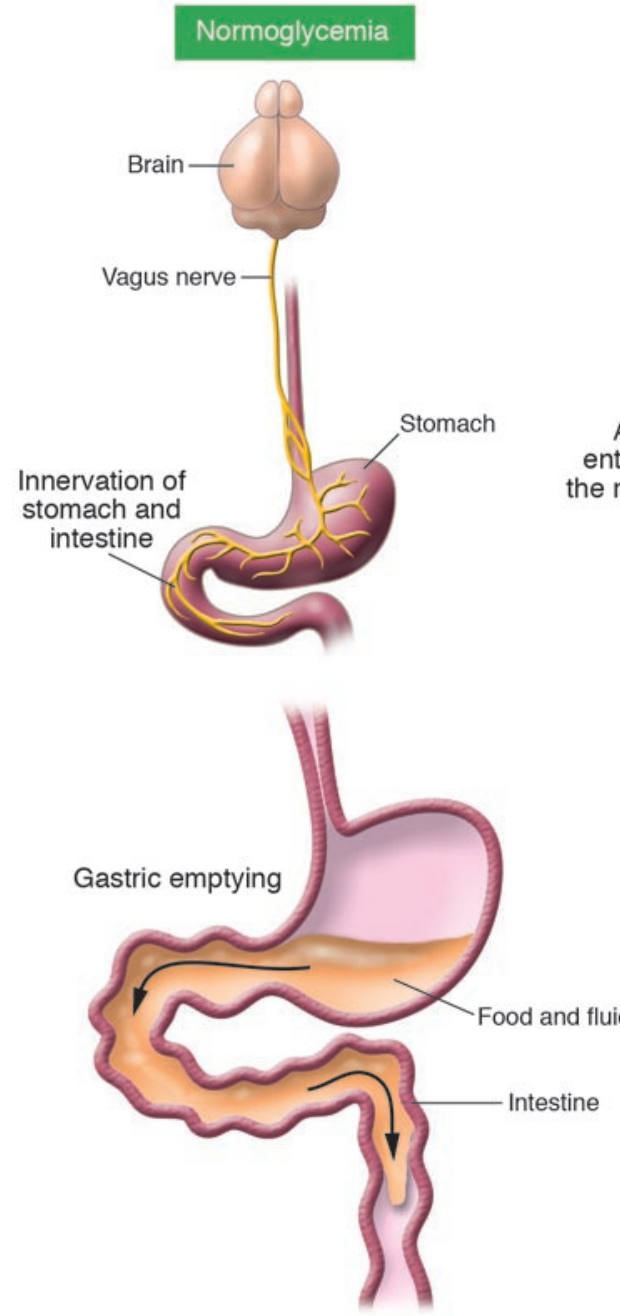

Intestinal transit
Hyperglycemia

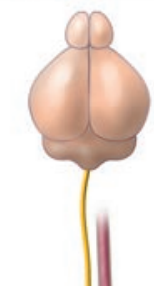

Decreased

Akt-mediated survival signaling

Apoptosis of the myenteric plexus
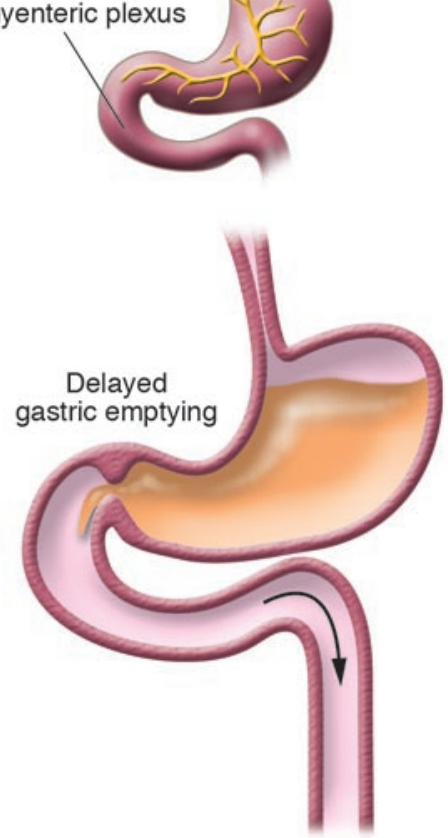

More rapid intestinal transit attributed to irreversible autonomic neuropathy, but it is known that the association between delayed gastric emptying and the presence of cardiovascular autonomic neuropathy is relatively poor (2). While this may partly be attributable to a current lack of tests to evaluate gastrointestinal autonomic dysfunction directly, it has now been established that acute changes in blood glucose concentration - both hyper- and hypoglycemia - have a marked, reversible, effect on gut motility (1). For example, in type 1 diabetic patients, gastric emptying is slower when the blood glucose concentration is elevated to $16-20 \mathrm{mmol} / \mathrm{l}$ when compared with euglycemia (4-8 mmol/l) (5), while in type 2 diabetes patients, cross-sectional studies indicate that the rate of gastric emptying also varies inversely with increasing glycemia (6). Conversely, acute hypoglyce$\mathrm{mia}(2.6 \mathrm{mmol} / \mathrm{l})$ induced by insulin accelerates gastric emptying in type 1 diabetes patients, even when emptying is slower than normal during euglycemia (7). Changes in blood glucose level within the physiological postprandial range $(4-8 \mathrm{mmol} / \mathrm{l})$ also affect gastric emptying (8), suggesting that gastrointestinal motor function is highly sensitive to the glycemic state and varies over relatively brief intervals.

At the same time, the rate of gastric emptying (and probably also patterns of small intestinal flow) has a substantial impact on carbohydrate absorption and the release of gut peptides, such as the incretin hormones glucagon-like peptide-1 (GLP-1) and glucose-dependent insulinotropic polypeptide (GIP), and is, accordingly, a major determinant of postprandial glycemic excursions - i.e., when gastric emptying is relatively slower, the initial glycemic and insulinemic responses to oral carbohydrate are less (1). This interrelationship of upper gut function with postprandial

\section{Figure 1}

The pathogenesis of gastrointestinal dysfunction in a diabetic mouse model, as proposed by Anitha et al. (18). Mice that are hyperglycemic as a result of STZ-induced diabetes have apoptosis of enteric neurons in the myenteric plexus, associated with delayed gastric emptying and rapid intestinal transit when compared with control mice. Activity of the PI3K/Akt pathway is decreased in the diabetic mice, which implies an impairment of retrograde axonal transport of neurotropic factors. Both neuronal apoptosis and disordered gastrointestinal motility are prevented in mice that overexpress GDNF.

glycemia (the chicken and the egg) is a concept of profound relevance to management strategies for diabetes, given that postprandial glycemia is probably the major determinant of "average" glycemic control, as assessed by glycated hemoglobin, the traditional marker of the risk of diabetic complications (i.e., retinopathy, neuropathy, and nephropathy) (9). Interventions that slow gastric emptying are likely to benefit glycemic control in type 2 diabetes patients managed by diet or oral hypoglycemic drugs, who have impaired early, but intact late, postprandial insulin responses, while prokinetic drugs may allow more predictable entry of carbohydrate into the small intestine in type 1 diabetes patients and thereby optimize the coordination between glucose absorption and the action of exogenous insulin. Slowing of gastric emptying is likely to contribute to the efficacy of the GLP-1 agonist exenatide and the amylin analog pramlintide in reducing glycated hemoglobin in diabetes; both drugs have recently been approved for use in the United States.

\section{Clues to the pathogenesis of disordered motility in animal models of diabetes}

To explain the disturbed gastrointestinal motility associated with diabetes, investigators have turned to animal models, primarily in search of evidence for gastrointestinal autonomic neuropathy. Diabetic rodents exhibit morphological changes involving axons and dendrites in the extrinsic autonomic nerves supplying the gut, particularly the distal segments of axons and nerve terminals, without much neuronal loss (10). Impaired retrograde transport of neurotrophic factors has been observed in the vagus nerve of streptozotocin-induced (STZ-induced) diabetic rats and may 
account for abnormalities of axonal growth and differentiation; recently, decreased activity of the PI3K pathway was implicated in the pathogenesis of impaired transport (11). Importantly, the axonopathy observed in the autonomic nerves of diabetic rodents is reversible with improved glycemic control, induced by exogenous insulin or pancreatic islet transplantation (12).

While the extrinsic autonomic nerves allow for central modulation of gut function, it is the enteric nervous system, incorporating the submucus and myenteric plexuses, that coordinates gastrointestinal motility more directly. In STZ-induced diabetic rats, there is a reduction in the number of myenteric neurons in both the stomach (13) and the colon (14); the latter is evident within 7 days of the onset of diabetes. The interstitial cells of Cajal (ICCs) play a central role in coordinating gut motility, generating an electrical slow wave that determines the rhythmicity of smooth muscle contraction. In the NOD mouse, in which gastric emptying is delayed, the number of gastric ICCs is markedly diminished (15). In rodent models of diabetes, neurotransmission is also impaired, with apparently selective loss of neuronal NOS expression in the gastric myenteric plexus (16), associated with delayed gastric emptying that is reversed by both the phosphodiesterase inhibitor sildenafil and insulin (16). Dysfunction of the autonomic/enteric nervous system in diabetes and impairment of the PI3K pathway in particular could potentially result from hyperglycemia (with its attendant oxidative stress), insulin deficiency (insulin activates PI3K and is antiapoptotic), and/or accumulation of advanced glycation end products (11). Horvath et al. recently reported that deficiency of insulin and IGF-1, as opposed to hyperglycemia per se, accounts for the depletion of gastric ICCs in the NOD mouse (17).

In this issue of the JCI, Anitha et al. report that hyperglycemia induces apoptosis of rodent enteric neurons in culture in association with impaired activity of the PI3K pathway (18) (Figure 1). Apoptosis was prevented by glial cell line-derived neurotrophic factor (GDNF). STZ-induced diabetic mice also displayed apoptosis in the myenteric plexus, which specifically involved nitrergic (inhibitory) neurons, and had delayed gastric emptying and more rapid intestinal transit than controls. Again, the PI3K pathway was implicated, and the changes were prevented in transgenic mice that overexpressed GDNF. These observations imply that hyperglycemia is responsible for apoptosis, but only allow speculation as to the mechanisms by which high glucose concentrations suppress PI3K activity. Furthermore, the potential contribution of insulin deficiency to the loss of enteric neurons was not examined, and while a means of preventing neuronal loss was demonstrated, the reversibility of this loss was not.

\section{Translation of animal findings to human diabetes}

How applicable are insights derived from rodent and other animal models to human diabetes? The available information in humans is limited by the relatively small number of studies and the substantial logistical difficulties in obtaining tissue and controlling for variables, such as the type and duration of diabetes and the adequacy of glycemic control. Early studies reported no evidence of morphological changes in the vagus nerve of patients with diabetic gastroparesis (19), although vagal conduction was reportedly slowed (20) and gastric secretion in response to sham feeding was diminished, suggestive of vagal dysfunction (21). Though limited to a small number of subjects, the most convincing observations to parallel rodent data are the depletion of ICCs in the stomach (22), jejunum (23), and colon (24) of patients with longstanding diabetes and gastroparesis; absence of gastric ICCs, in particular, appears to be associated with severe upper gastrointestinal symptoms and a poor response to treatment with gastric electrical pacing (22). There is also evidence for preferential loss of inhibitory neurotransmission in human diabetes, with impairment of meal-induced relaxation of the gastric fundus (25), excessive phasic contraction of the pylorus (26), and abnormal bursts of small intestinal contractile activity (27). However, in humans with diabetic gastroparesis as a group, there is also a reduction in antral contractions and impaired antroduodenal coordination, while an increase in pyloric activity is not invariably observed (28). One group has reported a gastric myopathic process in patients with severe diabetic gastroparesis (29). Moreover, attempts to accelerate gastric emptying with sildenafil in diabetic gastroparesis have hitherto been disappointing (30). A heterogeneous mixture of structural and functional neuromuscular abnormalities is, therefore, likely to account for disordered gut motility in human diabetes. Nor is it likely that there is a single underlying cause of dysfunction. The relative contributions of insulin deficiency or resistance versus hyperglycemia have not been clearly differentiated. Furthermore, the influence of chronic, as opposed to acute, hyperglycemia has not been adequately characterized; gut motility is altered within minutes of the induction of acute hyperglycemia in humans, which is presumably too rapid to be accounted for by the apoptosis of enteric neurons, as described in this issue by Anitha et al. (18). More attention should be devoted to the potential role of variations in the humoral environment in both animal models and patients with diabetes, as opposed to a purely neuropathic model of gastrointestinal dysfunction. For example, deficiencies of IGF-1, amylin, GLP-1, and peptide YY may be relevant, as may absolute or relative glucagon excess (31).

Current treatment of gastrointestinal dysfunction in diabetes is nonspecific and by no means uniformly effective, and accordingly, studies that identify potential targets for new therapies are to be welcomed. Future priorities are to discriminate between the various humoral and neuropathic etiologies of impaired gut function and, perhaps most importantly, to translate insights derived from animal models to human diabetes.

Address correspondence to: Chris Rayner, Department of Medicine, Royal Adelaide Hospital, North Terrace, Adelaide, South Australia 5000, Australia. Phone: 61-88222-2916; Fax: 61-8-8223-3870; E-mail: chris.rayner@adelaide.edu.au.

1. Rayner, C.K., Samsom, M., Jones, K.L., and Horowitz, M. 2001. Relationships of upper gastrointestinal motor and sensory function with glycemic control. Diabetes Care. 24:371-381.

2. Horowitz, M., et al. 1991. Relationships between oesophageal transit and solid and liquid gastric emptying in diabetes mellitus. Eur. J. Nucl. Med. 18:229-234.

3. Keshavarzian, A., and Iber, F.L. 1986. Intestinal transit in insulin-requiring diabetics. Am. J. Gastroenterol. 81:257-260.

4. Bytzer, P., et al. 2001. Prevalence of gastrointestinal symptoms associated with diabetes mellitus: a population-based survey of 15,000 adults. Arch. Intern. Med. 161:1989-1996.

5. Fraser, R., et al. 1990. Hyperglycaemia slows gastric emptying in type 1 diabetes mellitus. Diabetologia. 30:675-680.

6. Horowitz, M., et al. 1989. Gastric and oesophageal emptying in patients with type 2 (non-insulin-dependent) diabetes mellitus. Diabetologia. 32:151-159.

7. Russo, A., et al. 2005. Insulin-induced hypoglycemia accelerates gastric emptying of solids and liquids in long-standing type 1 diabetes. J. Clin. Endocrinol. Metab. 90:4489-4495. 
8. Schvarcz, E., et al. 1997. Physiological hyperglycemia slows gastric emptying in normal subjects and patients with insulin-dependent diabetes mellitus. Gastroenterology. 113:60-66.

9. Ceriello, A. 2005. Postprandial hyperglycemia and diabetes complications: is it time to treat? Diabetes. $54: 1-7$

10. Schmidt, R.E. 2002. Neuropathology and pathogenesis of diabetic autonomic neuropathy. Int. Rev Neurobiol. 50:257-292.

11. Cai, F., and Helke, C.J. 2003. Abnormal PI3 kinase/ Akt signal pathway in vagal afferent neurons and vagus nerve of streptozotocin-diabetic rats. Brain Res. Mol. Brain Res. 110:234-244.

12. Schmidt, R.E., Plurad, S.B., Olack, B.J., and Scharp, D.W. 1983. The effect of pancreatic islet transplantation and insulin therapy on experimental diabetic autonomic neuropathy. Diabetes. 32:532-540.

13. Fregonesi, C.E., Miranda-Neto, M.H., Molinari, S.L., and Zanoni, J.N. 2001. Quantitative study of the myenteric plexus of the stomach of rats with streptozotocin-induced diabetes. Arq. Neuropsiquiatr. 59:50-53.

14. Furlan, M.M., Molinari, S.L., and Miranda Neto, M.H. 2002. Morphoquantitative effects of acute diabetes on the myenteric neurons of the proximal colon of adult rats. Arq. Neuropsiquiatr. 60:576-581.

15. Ordog, T., Takayama, I., Cheung, W.K., Ward, S.M and Sanders, K.M. 2000. Remodeling of networks of interstitial cells of Cajal in a murine model of diabetic gastroparesis. Diabetes. 49:1731-1739.
16. Watkins, C.C., et al. 2000. Insulin restores neuronal nitric oxide synthase expression and function that is lost in diabetic gastropathy. J. Clin. Invest. 106:373-384

17. Horvath, V.J., Vittal, H., and Ordog, T. 2005. Reduced insulin and IGF-I signaling, not hyperglycemia, underlies the diabetes-associated depletion of interstitial cells of Cajal in the murine stomach. Diabetes. 54:1528-1533.

18. Anitha, M., et al. 2006. GDNF rescues hyperglycemia-induced diabetic enteric neuropathy through activation of the PI3K/Akt pathway. J. Clin. Invest. 116:344-356. doi:10.1172/JCI26295.

19. Yoshida, M.M., Schuffler, M.D., and Sumi, S.M. 1988. There are no morphologic abnormalities of the gastric wall or abdominal vagus in patients with diabetic gastroparesis. Gastroenterology. 94:907-914.

20. Tougas, G., Hunt, R.H., Fitzpatrick, D., and Upton, A.R. 1992. Evidence of impaired afferent vagal function in patients with diabetes gastroparesis. Pacing Clin. Electrophysiol. 15:1597-1602.

21. Hosking, D.J., Moody, F., Stewart, I.M., and Atkinson, M. 1975. Vagal impairment of gastric secretion in diabetic autonomic neuropathy. Br. Med. J. 2:588-590.

22. Forster, J., et al. 2005. Absence of the interstitial cells of Cajal in patients with gastroparesis and correlation with clinical findings. J. Gastrointest. Surg. 9:102-108.

23. He, C.L., et al. 2001. Loss of interstitial cells of cajal and inhibitory innervation in insulin-dependent diabetes. Gastroenterology. 121:427-434

24. Nakahara, M., et al. 2002. Deficiency of KIT-positive cells in the colon of patients with diabetes mellitus. J. Gastroenterol. Hepatol. 17:666-670.

25. Samsom, M., et al. 1998. Proximal gastric motor activity in response to a liquid meal in type 1 diabetes mellitus with autonomic neuropathy. Dig. Dis. Sci. 43:491-496

26. Mearin, F., Camilleri, M., and Malagelada, J.R. 1986. Pyloric dysfunction in diabetics with recurrent nausea and vomiting. Gastroenterology. 90:1919-1925.

27. Camilleri, M., and Malagelada, J.R. 1984. Abnormal intestinal motility in diabetics with the gastroparesis syndrome. Eur. J. Clin. Invest. 14:420-427.

28. Fraser, R., Horowitz, M., Maddox, A., and Dent, J. 1993. Organization of antral, pyloric and duodenal motility in patients with gastroparesis. J. Gastrointest. Motil. 5:167-175.

29. Ejskjaer, N.T., et al. 1999. Novel surgical treatment and gastric pathology in diabetic gastroparesis. Diabet. Med. 16:488-495.

30. Dishy, V., et al. 2004. The effect of sildenafil on gastric emptying in patients with end-stage renal failure and symptoms of gastroparesis. Clin. Pharmacol. Ther. 76:281-286.

31. Young, A.A., and Edwards, G.L. 2004. Effects of diabetes mellitus on gastrointestinal function in animal models. In Gastrointestinal function in diabetes mellitus. M. Horowitz and M. Samsom, editors. John Wiley and Sons Ltd. Chichester, United Kingdom. 29-95

\title{
Insulin's effect on the liver: "Direct or indirect?" continues to be the question
}

\author{
Jean Girard \\ Institut Cochin, Département Endocrinologie, Métabolisme et Cancer, UMR 8104 CNRS, U 567 INSERM, Université René Descartes, \\ Faculté de Médecine, Paris, France.
}

\begin{abstract}
Previous studies suggest that insulin can inhibit hepatic glucose production (HGP) by both direct and indirect actions. The indirect effects include inhibition of glucagon secretion, reduction in plasma nonesterified fatty acid levels, reduction of the amount of gluconeogenic precursor supplied to the liver, and change in neural input to the liver. A study in this issue of the JCI demonstrates that, in overnight-fasted dogs, an acute, selective increase of portal insulin induces a rapid inhibition of HGP, and a 4-fold rise in head insulin level does not enhance the inhibition of HGP in response to portal insulin infusion (see the related article beginning on page 521). This study demonstrates that insulin's direct effects on the liver dominate the control of HGP. These data balance previous studies in mice that suggested that indirect effects of insulin via the hypothalamus are the primary determinant of HGP.
\end{abstract}

\section{Introduction}

For a long time it was believed that the inhibition of hepatic glucose production (HGP) by insulin resulted only from a

Nonstandard abbreviations used: HGP, hepatic glucose production.

Conflict of interest: The author has declared that no conflict of interest exists.

Citation for this article: $J$. Clin. Invest. 116:302-304 (2006). doi:10.1172/JCI27743. direct effect of the hormone on the liver. However, 2 observations challenged this view: (a) whereas insulin is a potent inhibitor of HGP in vivo, the hormone is relatively ineffective in vitro in rodent liver (1), suggesting that insulin primarily acts on an extrahepatic tissue; and (b) peripheral insulin infusion in humans and dogs is just as effective as intraportal insulin infusion in suppressing HGP (2-4), suggesting that insulin can inhibit HGP by both direct and indirect actions (reviewed in ref. 5).

\section{Indirect action of insulin on HGP}

The indirect effects of insulin on HGP could be explained by its actions on pancreatic $\alpha$ cells, adipose tissue, and skeletal muscles. Insulin inhibits glucagon secretion from pancreatic $\alpha$ cells, thereby decreasing $\operatorname{HGP}(6,7)$. Adipose tissue and muscles are exquisitely sensitive to the inhibitory effect of insulin on lipolysis and proteolysis. Insulin induces a decrease in the release of nonesterified fatty acids and glycerol from adipose tissue (8) and gluconeogenic precursors from skeletal muscles (9), thus causing a decrease in hepatic gluconeogenesis. More recently, insulin action on the brain has been demonstrated to play a role in the regulation of HGP (10): infusion of insulin in the third cerebral ventricle of rats reduces HGP. The blockade of insulin receptors in the rodent hypothalamus (by injection of antisense oligonucleotides that 